\title{
Interference Effects in Nanocrystalline Systems
}

\author{
A. LEONARDI, M. LEONI, and P. SCARDI
}

\begin{abstract}
Interference (cross-correlation) effects are present in the X-ray powder diffraction pattern of a polycrystalline aggregate. In an experimental diffraction pattern, the information is highly overlapped and can be confused with other effects. In this article, it is shown that the analysis of the patterns calculated from a cluster equilibrated via molecular dynamics simulation allows those effects to be separated. Extra intensity is observed, because of the presence of the grain boundaries contribution which is unexpectedly not that of an amorphous phase.
\end{abstract}

DOI: $10.1007 / \mathrm{s} 11661-012-1428-6$

(C) The Minerals, Metals \& Materials Society and ASM International 2012

\section{INTRODUCTION}

RECENT studies have shown how interference effects can be observed in the diffraction from polycrystalline systems when scattering domains are sufficiently small and textured. ${ }^{[1-5]}$ Besides loose nanocrystalline powders, where the above conditions can arise from special growth or coalescence phenomena, ${ }^{[6,7]}$ these effects are important also in polycrystalline aggregates as they can influence the overall properties of the system. ${ }^{[3]}$

Models have been proposed to describe the special condition when the diffracted intensity distributions from small and closely oriented domains overlap in Reciprocal Space (RS), thus affecting the observed powder patterns in different ways. ${ }^{[1-5]}$ This is especially visible for low Miller indices peak profiles, corresponding RS points of which are closer to the origin. Further insights have been provided by a few recent studies, where interference effects among the nanosized metallic domains were simulated using atomistic models and the Debye scattering equation (DSE $)^{[8,9]}$ to generate the corresponding powder patterns. ${ }^{[10,11]}$

In all of these cases, however, interference was related to the size and orientation of the domains without the consideration of the role of grain boundaries, which indeed contribute a considerable fraction to nano-scale materials (see, e.g., References 12 through 14). Models and simulations so far have treated nanocrystals as small (perfect) single crystals, without accounting for the boundary region and of the natural tendency of the system to achieve a minimum of energy. In the current study, we provide a preliminary understanding of the possible effects of the grain boundary (GB) on the diffraction patterns from systems made of small crystalline domains. In particular, it is shown that the signal from the GB region can be partly coherent with the bulk of the neighboring crystalline domains.

A. LEONARDI, Ph.D. Student, M. LEONI, Assistant Professor, and P. SCARDI, Professor, are with Department of Civil, Environmental and Mechanical Engineering, University of Trento, via Mesiano, 77,38123 Trento, Italy. Contact e-mail: paolo.scardi@unitn.it.

Manuscript submitted May 11, 2012.

Article published online September 18, 2012

\section{GENERATION OF THE NANO- POLYCRYSTALLINE MODEL}

A nano-polycrystalline cluster made of 50 grains of $\mathrm{Cu}$ was built via Constrained Voronoi Tessellation $\left(\mathrm{CVT}^{[15]}\right)$ in a cubic box of $260.28 \AA$ with PBCs (Figure 1). Using this technique, the size and shape of the grains were tuned to obtain a lognormal dispersion of normalized volumes (mean volume $352657 \AA^{3}$, standard deviation $=0.35$; the diameter of a sphere having the mean volume is $c a$. $70 \AA$ ). The distribution character was confirmed by the Kolmogorov-Smirnov test at 5 pct significance level. A $f c c$ copper structure (cell parameter $a=3.615 \AA$ ) was placed in the grains, eliminating those atoms on the boundary whose distances are below $85 \mathrm{pct}$ of the minimum ideal value $(3.615 / \sqrt{8}){ }^{[16]}$ The distribution of misorientation angles agree with the MacKenzie result ${ }^{[17]}$ associated to a randomly textured material.

The system was equilibrated at $100 \mathrm{~K}\left(-173.15^{\circ} \mathrm{C}\right)$ under NPT conditions using molecular dynamics and the Embedded Atom Method potential for $\mathrm{Cu}^{[18,19]}$ The LAMMPS $\operatorname{code}^{[20]}$ was employed.

Once stationary conditions were reached, a sequence of 100 independent frames was collected each $1 \mathrm{ps}$. The position of each atom (labeled with the grain ID) was averaged over the collected dataset in order to remove any dynamic (thermal) component. ${ }^{[21]}$ A powder diffraction pattern was computed via DSE for each of those averaged datasets; the PowDOG ${ }^{[9]}$ code running on a cluster of NVIDIA GPUs was employed. Because of the averaging, these diffraction patterns contain only information on size and static displacements: local distortion, non-crystalline features, and defects contributions to line profile broadening can therefore be more easily separated.

\section{RESULTS AND DISCUSSION}

When the DSE is used for the cluster as a whole, the resulting powder pattern includes correlation effects between all atoms, irrespective of whether they belong to same or different grains, to the core or to GB regions. The range of the powder pattern comprising the (111) 


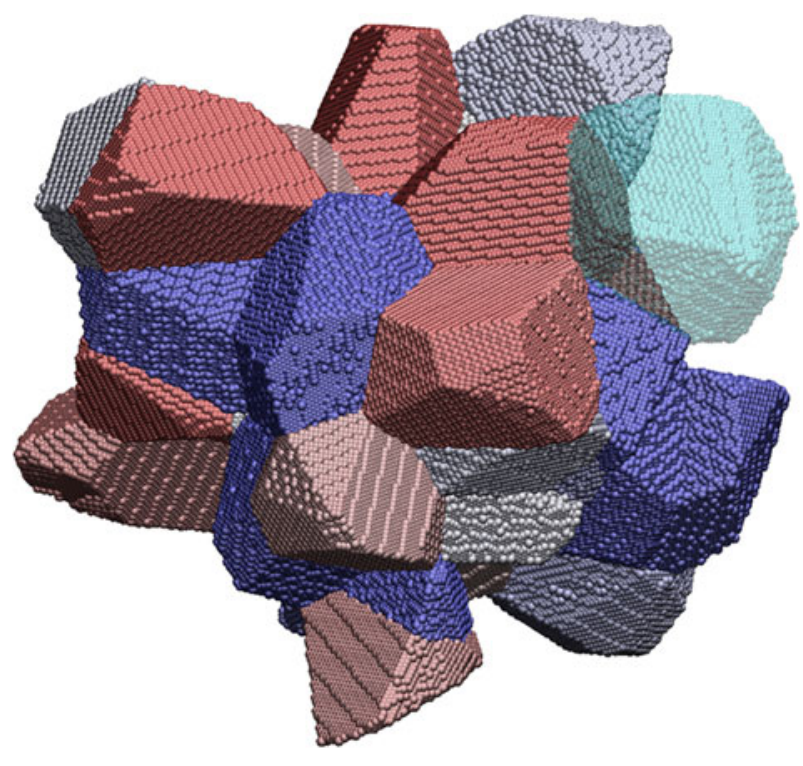

Fig. 1-Cluster employed for the MD simulation.

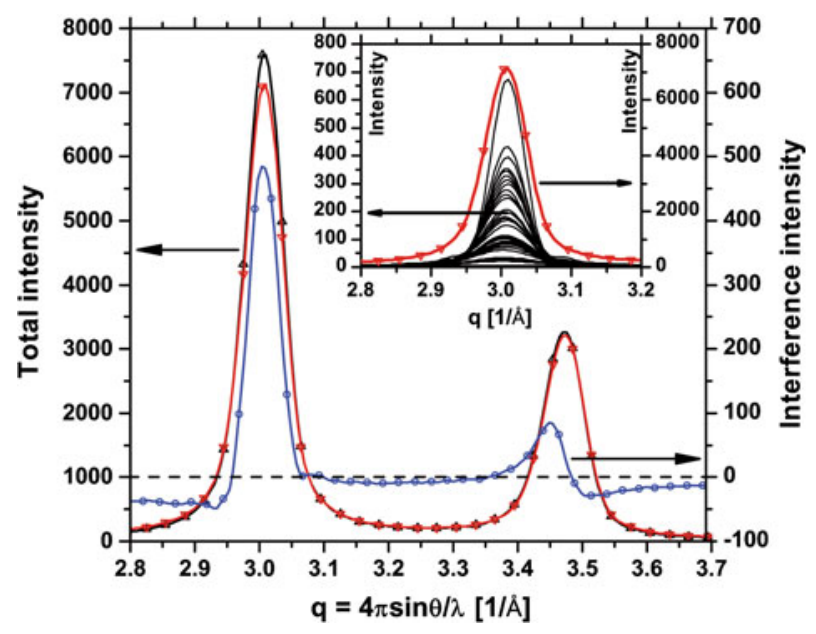

Fig. 2-Portion of the X-ray powder diffraction pattern of the cluster (black, up triangles) compared with the corresponding sum of the patterns from the component grains (red, down triangles): the difference coming from the grain-grain interference is evidenced (IEP-blue, circles). As an example, the inset shows the (111) peaks from the various grains (black lines) and their sum (red, down triangles) (Color figure online).
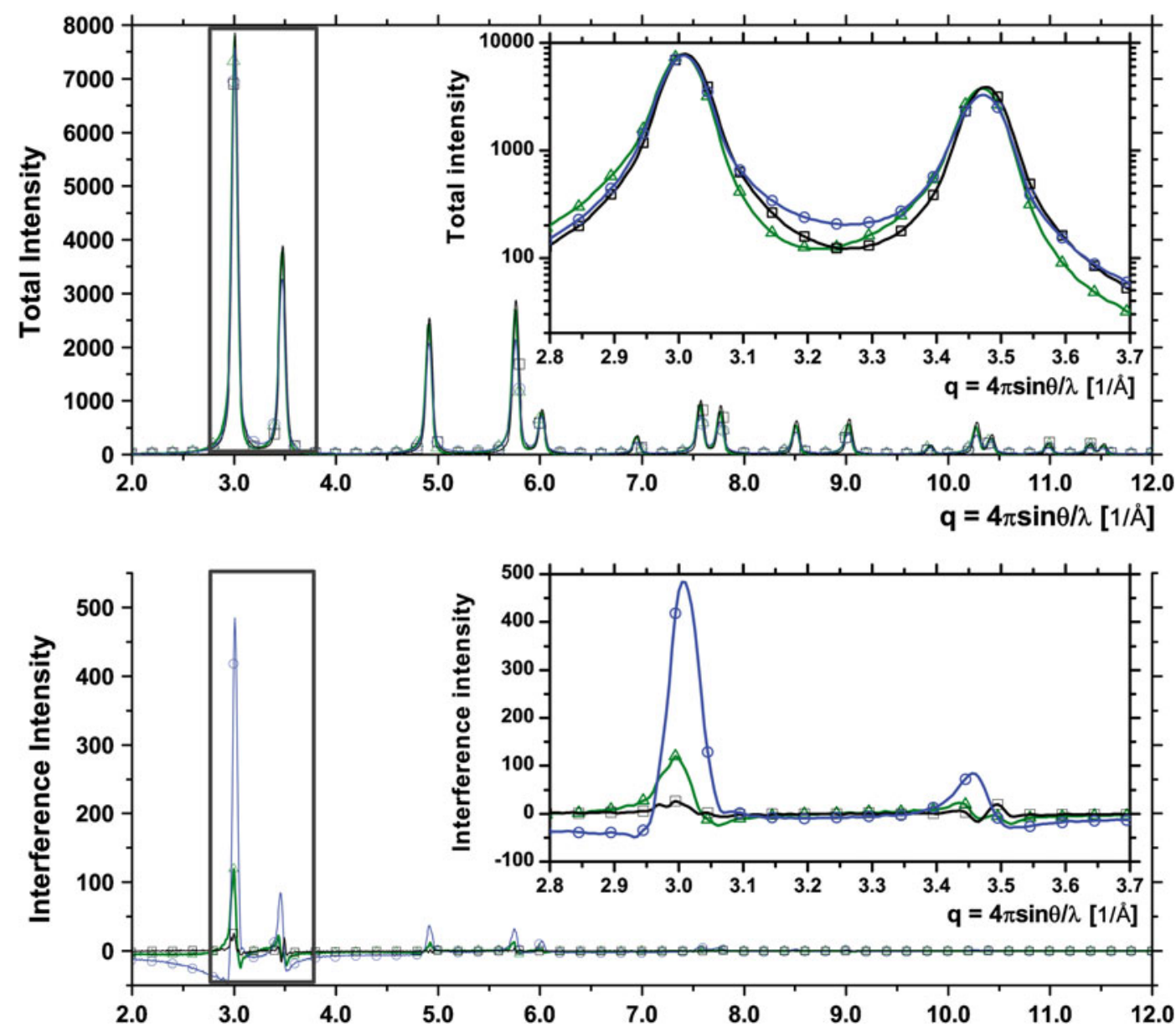

Fig. 3-In the upper plot, X-ray powder diffraction pattern for the cluster calculated for the starting microstructure (black squares), after energy minimization (green upper triangles) and after a long equilibration (blue, circles). The rectangle indicates the portion of the pattern considered in Fig. 2. In the lower plot, IEPs corresponding to the three cases are presented in the upper part. See text for details (Color figure online). 

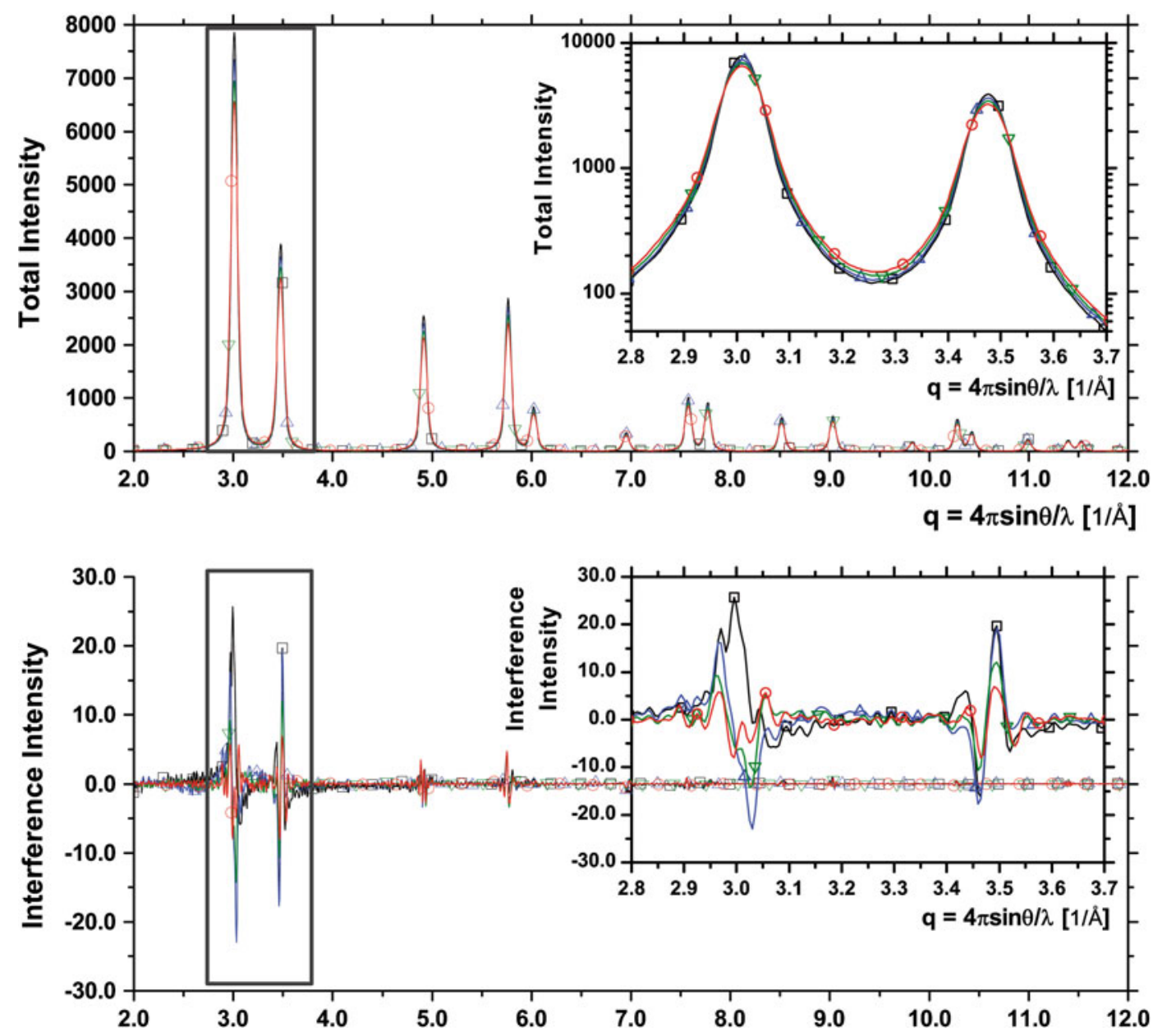

Fig. $4-$ GB contribution. In the upper part, the pattern of the "perfect" cluster (black squares) together with those obtained by removing 1 layer (CL1 - blue upper triangles), 2 layers (CL2 - green, down triangles), and 3 layers (CL3 - red, circles). The rectangle indicates the portion of the pattern considered in Fig. 2. In the lower plot, IEPs corresponding to the three cases are presented in the upper part. See text for details (Color figure online).

and (200) copper peaks is shown in Figure 2 for the equilibrated cluster, together with the corresponding pattern obtained by summing up all DSE patterns for each separate grain. The process in detail is shown in the inset.

To study the interference effects, we can use a plot of the difference between those contributions, i.e., between the pattern of the cluster and the sum of the patterns of the contributing grains (interference effects plot, IEP). As shown in this and in the following figures, the effect is clearly visible and is peaked around the Bragg positions.

To further understand the origin of the interference component, it is useful to compare the DSE pattern for whole cluster and the sum of separate grains in three distinct cases ( $c f$. Figure 3): (i) for the starting microstructure, just after the atom filling step, which is then made of "perfect" crystals; (ii) after the energy minimization step; and (iii) after a MD trajectory sufficiently long to equilibrate the microstructure (same as in Figure 2). The main effect of the energy minimization is a shift of Bragg's peaks with respect to case (i), caused by the expansion of the cells that better fill the intergranular regions. The MD equilibration has a more complex effect (still driven by energy minimization), leading to a reorganization of the atoms in the GB region. A diffuse scattering component-especially visible in the region between the main peaks - arises at the expenses of the Bragg's component, as an effect of the disordered structure of the GB region in the equilibrated model (iii).

The IEPs in the lower part of Figure 3 show that interference effects are quite small for (i), in agreement with the recent study by Gelisio and Scardi. ${ }^{[1]}$ Quite surprisingly, the interference component increases with energy minimization (ii) and even further after MD equilibration (iii). As already pointed out, the latter step mostly rearranges atoms in the GB region, with negligible grain rotations; it is therefore clear that the main contribution to interference comes from the GB.

The contribution of the GB is made evident by the following analysis. The powder pattern of the "perfect" crystalline cluster (case (i) above) can be compared with that of the same cluster after removing all atoms with a coordination lower than 12 (CL1), or after removing all atoms the first (CL2) or the second (CL3) neighbors of which have coordination lower than 12 . This corresponds 

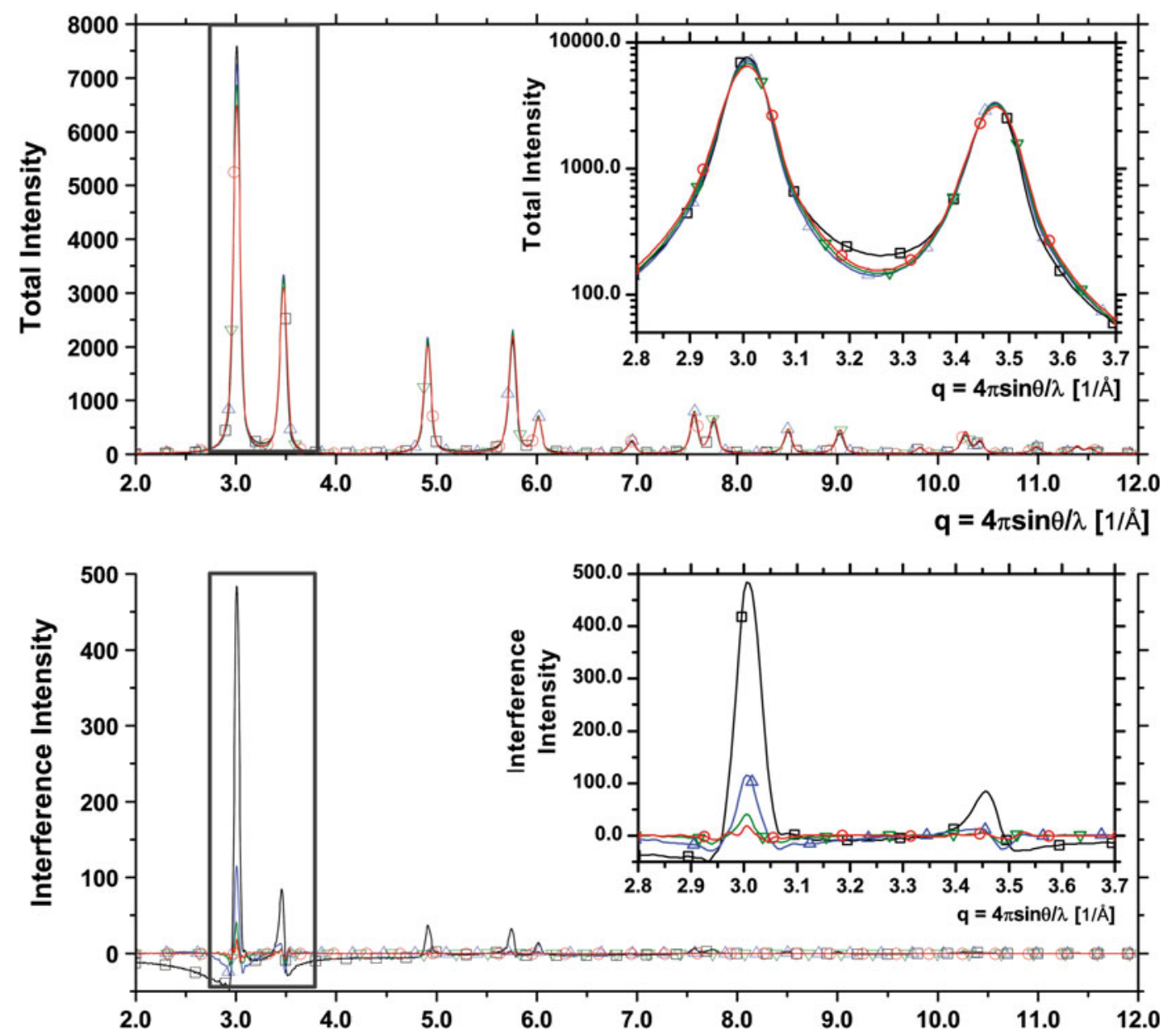

Fig. 5- GB contribution. In the upper part, the pattern of the equilibrated cluster (black squares) together with those obtained by removing 1 layer (CL1 - blue upper triangles), 2 layers (CL2 - green, down triangles), and 3 layers (CL3 - red, circles). The rectangle indicates the portion of the pattern considered in Fig. 2. In the lower plot, IEPs corresponding to the three cases are presented in the upper part. See text for details (Color figure online).

to progressively remove (peel off) atoms, layer by layer from each grains, thus removing the GB region. This is shown in Figure 4 for the starting perfect crystal microstructure (i). In this case, as already discussed, the interference effect is small; the further decreases observed in CL1, CL2, CL3 are mostly due to the volume decrease, which is also the reason for the broadening of the Bragg's peaks (easily visible in the top right inset).

The same analysis is shown in Figure 5 for the MDequilibrated cluster (iii). In this case, we can observe that the first removal step (CL1), contrary to what just observed for (i), gives a sharpening of the Bragg's peaks, as a consequence of the removal of the diffuse scattering component caused by the GB. It is also apparent (bottom right inset) that interference effects are strongly reduced in CL1 and comparable with (i) for CL2 and CL3. This further validates the results on the role of the GB in the interference phenomenon.

Finally, Figure 6 shows the DSE calculation obtained by considering just (a) CORE-CORE correlations between atoms in the core of the grains only (i.e., all GB atoms are excluded); (b) BOUNDARY-BOUNDARY correlations between GB atoms only; and (c) CORE-BOUNDARY, correlations between GB atoms and atoms of the grains. The analysis is then repeated for the CL1, CL2, and CL3 clusters, so that the sum of (a) to (c) gives the corresponding CL1, CL2, and CL3 patterns shown in Figure 5.

The first group (a) gives powder patterns typical of $f c c$ structures, with a progressive broadening of the Bragg's peaks caused by the decrease in grain size, from CL1 to CL3. The pattern produced by GB atoms (b) gives a main diffuse scattering component with a Bragg's component which is only visible for low Miller indices; the latter effect is due to the high disorder in the GB that enhances line broadening with the scattering vector until it cannot be distinguished from the diffuse scattering component. For increasing layer removal, the Bragg's component sharpens, as it includes more correlations between distant atom couples. The last group (c) shows that the correlation between GB atoms and grain cores also consists of a diffuse scattering component, but the Bragg's signal is stronger than that in (b).

An experimental validation of the arguments presented here is difficult to obtain in practice, as it is impossible to separate in this easy way the constituents of a diffraction pattern. The results are therefore tightly bound to the performance of the MD (thus to the choice 

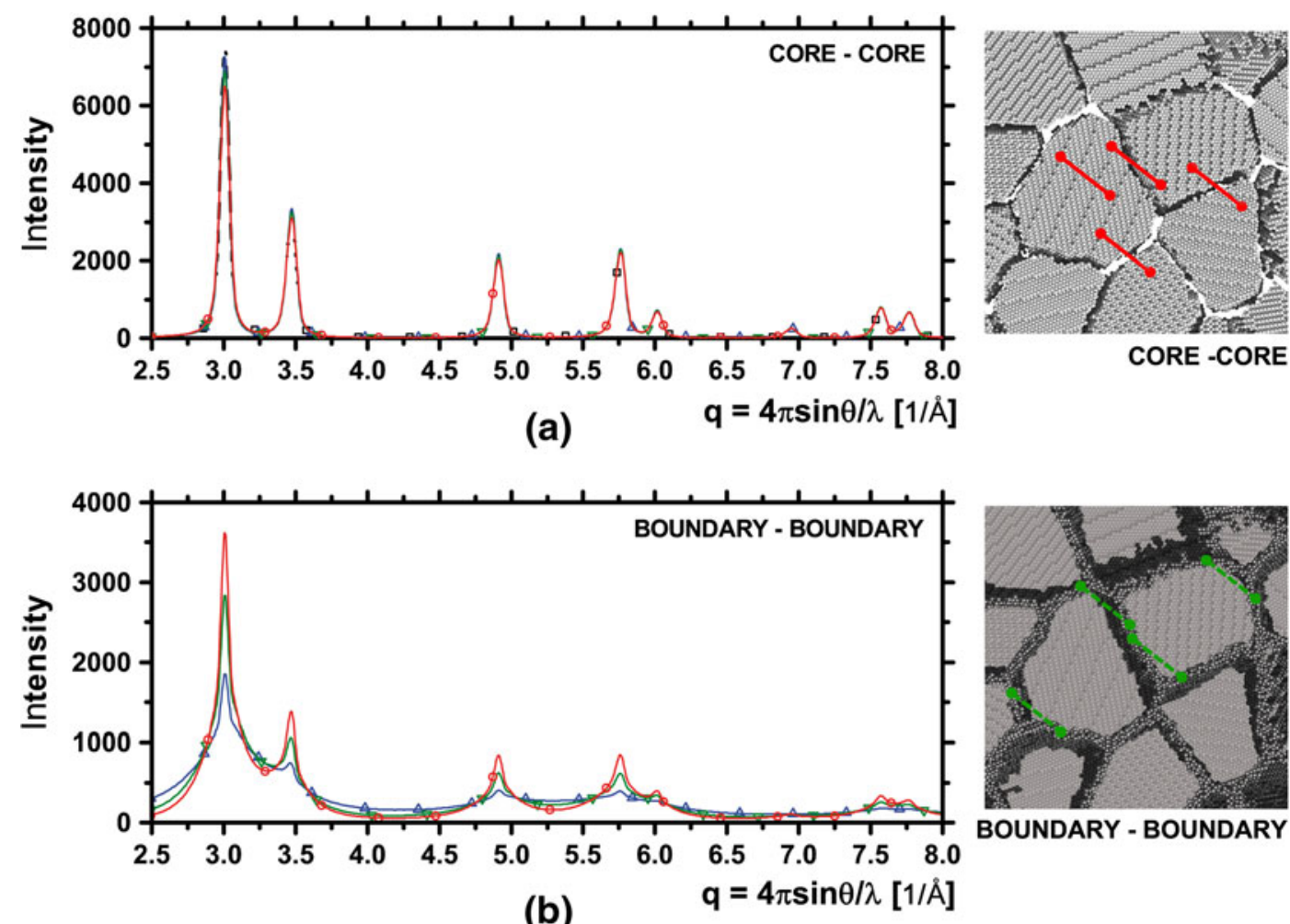

(b)
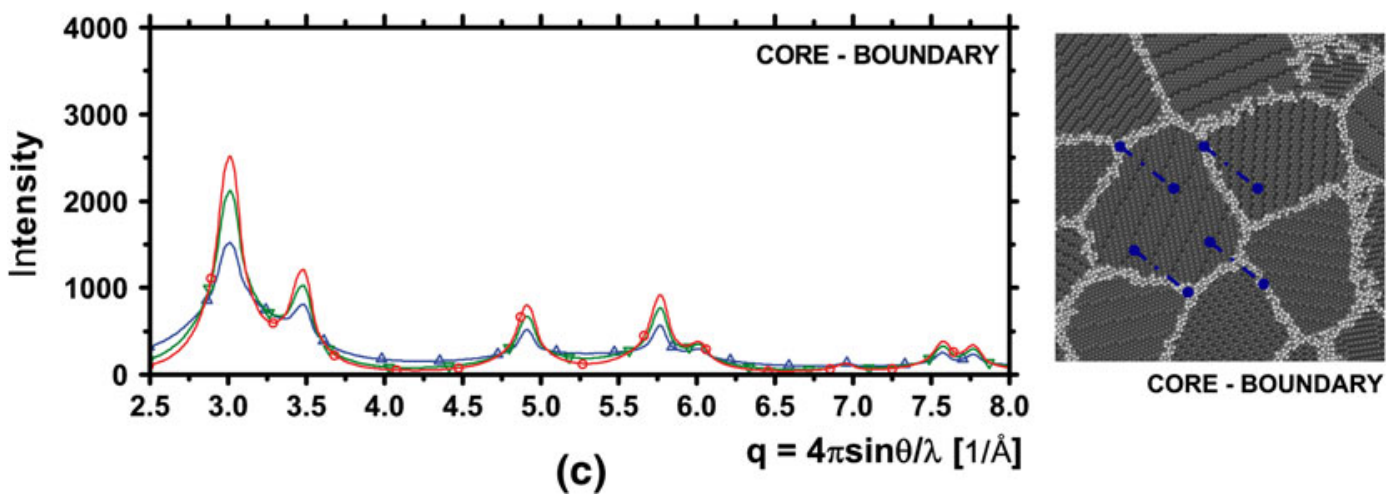

Fig. 6-DSE calculations obtained by considering just (a) CORE-CORE, correlations between atoms in the core of the grains, $(b)$ BOUNDARYBOUNDARY, correlations between GB atoms only; (c) CORE-BOUNDARY, correlations between GB atoms and atoms of the grains. In the three cases, plots represent the CL1 (blue upper triangles), CL2 (green, lower triangles), and CL3 (red circles) clusters (Color figure online).

of microstructure and potentials) and to its appropriateness in representing the microstructure of the cluster analyzed here. MD is known to provide a realistic representation of a material when limited to equilibration effects like those shown here. The use of a particular MD model does not change the fact that GBs are clearly different than the cores: it is therefore likely that interference effects due to the presence of the GB are present in a real microstructure as well.

\section{CONCLUSIONS}

Molecular dynamics applied to a nano-polycrystalline cluster allowed the various contributions to the diffraction pattern (static, thermal, defects, grain boundaries, etc.) to be singled out. In particular, it was shown in this study that the diffraction pattern of a cluster is not the mere sum of the contributions from all grains, but additionally diffuse and broadening effects are present. These effects are due to the interference of the grains in the cluster, and in particular they are mainly caused by the particular structure of grain boundaries.

\section{ACKNOWLEDGMENTS}

The authors wish to thank Mr Luca Gelisio for his useful discussions and providing support with the PowDOG software. 


\section{REFERENCES}

1. D. Rafaja, V. Valvoda, J. Kub, K. Temst, M.J. Van Bael, and Y. Bruynseraede: Phys. Rev. B, 2000, vol. 61, pp. 16144-53.

2. D. Rafaja, V. Klemm, G. Schreiber, M. Knapp, and R. Kužel: $J$. Appl. Crystallogr., 2004, vol. 37, pp. 613-20.

3. D. Rafaja, A. Poklad, V. Klemm, G. Schreiber, D. Heger, M. Śíma, and M. Dopita: Thin Solid Films, 2006, vol. 514, pp. 240-49.

4. D. Rafaja, C. Wüstefeld, M. Dopita, M. Ržika, V. Klemm, G. Schreiber, D. Heger, and M. Síma: Surf. Coat. Technol., 2007, vol. 201, pp. 9476-84

5. S. Förster, A. Timmann, C. Schellbach, A. Frömsdorf, A Kornowski, H. Weller, S.V. Roth, and P. Lindner: Nat. Mater., 2007, vol. 6, pp. 888-93.

6. S. Link, C. Burda, Z.L. Wang, and M.A. El-Sayed: J. Chem. Phys., 1999, vol. 111, pp. 1255-64.

7. P. Scardi, M. Leoni, M. Müller, and R. Di Maggio: Mater. Sci. Eng. A, 2010, vol. 528, pp. 77-82.

8. P. Debye: Ann. Phys., 1915, vol. 351, pp. 809-823.

9. L. Gelisio, C.L. Azanza Ricardo, M. Leoni, and P. Scardi: J. Appl. Crystallogr., 2010, vol. 43, pp. 647-53.
10. K.R. Beyerlein, R.L. Snyder, M. Li, and P. Scardi: Philos. Mag., 2010, vol. 90, pp. 3891-3905.

11. L. Gelisio and P. Scardi: J. Nanosci. Nanotechnol., 2012, vol. 12, in press.

12. M.A. Krivoglaz: X-Ray and Neutron Diffraction in Nonideal Crystals, Springer-Verlag, Berlin, 1996.

13. R.I. Barabash and P. Klimanek: J. Appl. Crystallogr., 1999, vol. 32, pp. 1050-59.

14. L.E. Levine, P. Geantil, B.C. Larson, J.Z. Tischler, M.E. Kassner, W. Liu, M.R. Stoudt, and F. Tavazza: Acta Mater., 2011, vol. 59, pp. $5803-11$.

15. D. Gross and M. Li: Appl. Phys. Lett., 2002, vol. 80, pp. 746-49.

16. T. Xu and M. Li: Phil. Mag., 2010, vol. 90, pp. 2191-2222.

17. J.K. Mackenzie: Biometrika, 1958, vol. 45, pp. 229-240.

18. M.S. Daw and M.I. Baskes: Phys. Rev. Lett., 1983, vol. 50 (17), pp. $1285-88$.

19. S.M. Foiles, M.I. Baskes, and M.S. Daw: Phys. Rev. B, 1986, vol. 33, pp. 7983-91.

20. S.J. Plimpton: J. Comput. Phys., 1995, vol. 117, pp. 1-19.

21. A. Leonardi, K.R. Beyerlein, T. Xu, M. Li, M. Leoni, and P. Scardi: Z. Kristall. Proc., 2011, vol. 1, pp. 37-42. 